\title{
Effects of caffeine and a tea flavanoid on Monacrosporium ambrosium, ectosymbiote of shot-hole borer beetle of tea
}

\author{
Naleen B. Jayaratna, D. Nedra Karunaratne and N. Savitri Kumar \\ Department of Chemistry, Faculty of Science, University of Peradeniya, Peradeniya.
}

Keywords: Caffeine, ectosymbiote, flavanoid, Monacrosporium ambrosium, tea

Shot-hole borer (SHB) beetle Euwallacea fornicatus (Xyleborus fornicatus) Eichh. (Coleoptera: Scolytidae) is the most serious insect pest of tea (Camellia sinensis L.) plantations in Sri Lanka. Caffeine, the major alkaloid found in tea, inhibits the growth of both Monacrosporium ambrosium, ectosymbiote of SHB and the SHB beetle, in laboratory culture media ${ }^{1,2}$. Caffeine contents of two tea cultivars that are resistant (TRI 2023) and susceptible (TRI 2025) to SHB infestation ${ }^{3}$, indicated that there was an increase in caffeine content after SHB infestation of both tea cultivars, the increase being higher in the more susceptible tea cultivar ${ }^{4}$. However, the two organisms are able to develop within tea stems that have a caffeine concentration which is higher than the concentration that inhibited the development of M. ambrosium and SHB beetles in laboratory culture media.

Flavan-3-ols and their gallate esters (catechins) may constitute up to $30 \%$ of young tea shoots. Epigallocatechin gallate (EGCG), the major phenolic compound found in tea, constitutes $9-13 \%$ of the dry weight of young tea shoots ${ }^{5}$. The present study was carried out to determine whether EGCG modulates the effect of caffeine on M. ambrosium in laboratory culture media.

The effect of caffeine and EGCG on M. ambrosium was studied by determining the fungal colony diameter, sporulation and spore germination on solid agar media and mycelial mass in liquid culture media respectively, into which varying amounts of these two phytochemicals were incorporated. The ambrosia fungus was isolated ${ }^{1}$ from a surface sterilized adult female SHB removed from a gallery in a tea stem. The eight types of media (1-8), were prepared ${ }^{2}$ in Cook's 2 quarter strength agar medium: 1. Control, 2. Caffeine 500 ppm, 3. EGCG $500 \mathrm{ppm}$, 4. Caffeine $500 \mathrm{ppm}+$ EGCG $500 \mathrm{ppm}$, 5. Caffeine $1000 \mathrm{ppm}, 6$. Caffeine $1000 \mathrm{ppm}+$ EGCG $500 \mathrm{ppm}, 7$. Caffeine $250 \mathrm{ppm}$ and 8. Caffeine 250 ppm + EGCG 500 ppm. A fungal disc, cut from a well grown colony of M. ambrosium was placed in the center of a Petri dish containing each of the above media and the diameter of the fungal colony was measured daily for five days (3 replicates). Sporulation was studied using a spore suspension prepared from fungal discs (30, $8 \mathrm{~mm}$ diameter) cut from a fungal colony grown in each type of medium. These were mixed with sterile water (4 mL, Vibromix, $30 \mathrm{~s})$, filtered through sterilized glass wool and the number of spores in each sample was counted using a hemocytometer (3 replicates). Spore germination was studied using fungal mycelia scraped from the tops of fungal colonies growing in different media. These were dissolved in sterile water $(5 \mathrm{~mL})$, shaken (Vibromix $30 \mathrm{~s}$ ), filtered through glass wool and centrifuged (3 $\mathrm{min}, 3000 \mathrm{rpm})$. The supernatant $(3 \mathrm{~mL}$ ) was removed; one drop of solution from the bottom of the tube was placed on a microscope slide and left aside in a moisture chamber for $9 \mathrm{~h}$. The number of germinated spores in 10 microscopic fields was counted. The percentage of spores that had germinated in each sample was calculated (3 replicates). Several experiments showed that sporulation did not occur in the presence of $1000 \mathrm{ppm}$ of caffeine. Therefore, sporulation and spore germination in media 5 and 6 containing $1000 \mathrm{ppm}$ of caffeine were not studied.

The effect of caffeine and EGCG on liquid cultures were studied by adding a spore suspension $(20 \mathrm{~mL}$, 
$5 \times 10^{3}$ spores $\left./ \mathrm{cm}^{2}\right)^{1}$ to a Czapek-dox liquid culture medium $(2000 \mathrm{~mL})^{4}$. Calculated amounts of caffeine and /or EGCG was added to each of twenty $250 \mathrm{~mL}$ conical flasks under sterile conditions to obtain concentrations

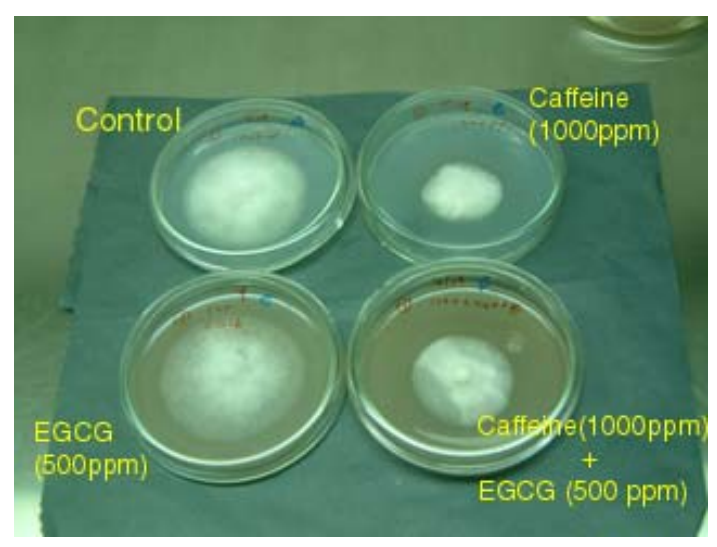

Figure 1: Control (1), EGCG 500 ppm (3) and Caffeine 1000 ppm (5) Caffeine 1000 ppm + EGCG 500 ppm (6) similar to the solid agar media 1, 2, 3, 4 and the liquid culture medium $(100 \mathrm{~mL})$ was added to each flask and left on a shaker for $5 \mathrm{~d}$. Fungal mycelia were separated and weighed (5 replicates).

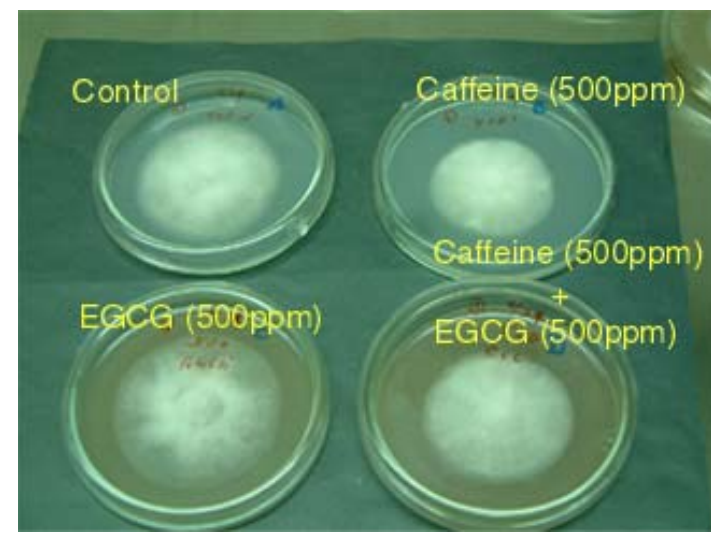

Figure 2: Control (1), Caffeine 500 ppm (2), EGCG 500 ppm (3) and affeine 500 ppm + EGCG 500 ppm (4)

Table 1: Effect of Caffeine and ECGC on M. ambrosium on laboratory culture media

\begin{tabular}{|c|c|c|c|c|c|}
\hline & & \multicolumn{4}{|c|}{ Growth Parameters- Percentage Reduction } \\
\hline \multicolumn{2}{|c|}{$\begin{array}{l}\text { Medium (No.) } \\
\text { Treatment Parameters }\end{array}$} & $\begin{array}{c}\text { Fungal colony } \\
\text { diameter }\end{array}$ & Sporulation & Spore germination & Fungal mass \\
\hline 1 & Control & 0.0 & 0.0 & 0.0 & 0.0 \\
\hline 2 & Caffeine 500 ppm & 17.5 & 85.0 & 55.0 & 23.6 \\
\hline 3 & EGCG 500 ppm & 0.0 & 5.0 & 36.0 & 56.0 \\
\hline 4 & Caffeine 500ppm + EGCG 500ppm & 15.0 & 90.0 & 39.0 & 58.0 \\
\hline 5 & Caffeine 1000 ppm & 42.0 & Not done & Not done & Not done \\
\hline 6 & Caffeine 1000ppm + EGCG 500 ppm & 37.0 & Not done & Not done & Not done \\
\hline 7 & Caffeine 250 ppm & 7.5 & 70 & 55.0 & Not done \\
\hline 8 & Caffeine 250ppm + EGCG 500 ppm & 5.0 & 70 & 59.0 & Not done \\
\hline
\end{tabular}

The percentage reduction in the diameter of the fungal colony in caffeine containing media $2,4,5,6(500 \mathrm{ppm}$ and $1000 \mathrm{ppm}$ caffeine), 7 and 8 (250 ppm caffeine) was significantly greater when compared to the control medium 1 (Figure 1) and the EGCG containing medium 3 (Table 1). Therefore, the presence of EGCG in the medium apparently does not have an effect on the diameter of the fungal colony while the percentage reduction in the diameter of the fungal colony was less in caffeine media which contained EGCG (media 4, 5 and 6). Therefore, EGCG modulates the inhibitory effect of caffeine on mycelial development on laboratory media.

The presence of EGCG did not significantly modify the effect of caffeine on sporulation. A pronounced reduction in sporulation was observed in the caffeine containing media when compared to sporulation in the control medium 1 as observed in our previous study ${ }^{4}$ (Table 1). Sporulation in medium 3 (with EGCG) was not significantly different from sporulation in the control medium 1 and the presence of EGCG did not significantly modify the effect of caffeine on sporulation (Table 1, media $7 \& 8$ ). A significantly higher number of spores germinated from mycelia grown in EGCG containing media 3 and 4, when compared with caffeine containing media 2, 7 and 8 . Therefore, the inhibitory effect of caffeine on spore germination was reduced in the presence of EGCG.

A significant reduction in the weight (56\% and 58\% respectively) of mycelia was observed in mycelia grown in the EGCG containing media 3 and 4 respectively 
(Table 1). A reduction in mycelial growth $(23.6 \%)$ was also observed in 2, but this difference was not significant when compared with the reduction of mycelial mass observed in the presence of EGCG (3 and 4). The pronounced effect of EGCG on mycelial mass is also clearly seen in Figures 1 and 2, which show that the fungal colony area is more diffuse and less dense in the EGCG containing medium 3 compared to control medium 1 , while the fungal colony is more compressed and dense in the caffeine containing media 2 and 5 .

Results discussed above were obtained after statistical analysis by one way ANOVA test using SAS computer software. The $p$ value was between $0.004-0.0001$ for all the experiments. According to the Duncan's Multiple Range test, groups that were significantly different were identified.

Polyphenols such as EGCG are known to have the ability to bind proteins and the restriction in fungal growth may therefore be due to inhibition of certain fungal enzymes ${ }^{6}$. Colonization of xylem vessels by the ambrosia fungus and the presence of globular structures with brown cell contents around the colonized region, reported by us during a previous study ${ }^{7}$ suggest that accumulation of tannins and fungistatic flavanols occurs after gallery formation by SHB attack and may produce an environment that is restrictive to intercellularly spreading pathogens. It is possible that flavanols such as EGCG and other catechins found in tea stems may be involved in a biochemical role to restrict the intercellular spreading of fungal mycelia, and thereby serve to limit the damage caused by SHB to tea stems.

\section{Acknowledgement}

The authors thank Prof. N.K.B. Adikaram, Department of Botany, University of Peradeniya, for many useful suggestions; the Tea Research Institute, Hantane substation, Kandy for cooperation and assistance and the National Science Foundation of Sri Lanka for research funding.

\section{References}

1. Kumar N.S., Hewavitharanage P. \& Adikaram N.K.B. (1995). Attack on tea by Xyleborus fornicatus: inhibition of the symbiote, Monacrosporium ambrosium, by caffeine. Phytochemistry 40: 1113-1116.

2. Hewavitharanage P., Karunaratne S. \& Kumar N.S. (1999). Effect of caffeine on shot-hole borer beetle (Xyleborus fornicatus) of tea (Camellia sinensis). Phytochemistry 51: $35-41$.

3. Calnaido D. \& Kanapathipillai P. (1967). Tolerance and susceptibility of tea clones to shot hole borer beetle, Xyleborus fornicatus Eichh. Coleoptera:Scolytidae. Tea Science 38: 275-281.

4. Karunaratne W.S., Kumar V. \& Kumar N.S. (2008). Shothole borer infestation of tea: ovicidal effect of caffeine. Science and Culture 74(1-2): 59-64.

5. Arulpragasam P.V. (1992). Disease Control in Asia In: Tea Cultivation and Consumption (Eds. K.C. Wilson and M.N. Clifford), pp 555-556, Chapman and Hall, London, UK.

6. Haslam E. (1998). Practical Polyphenolics. From Structure to Molecular Recognition and Physiological Action. pp. 323-325 Cambridge University Press, UK.

7. Kumar N.S., Hewavitharanage P. \& Adikaram N.K.B. (1998). Histology and fungal flora of shot-hole borer beetle (Xyleborus fornicatus) galleries in tea (Camellia sinensis). Journal of the National Science Council of Sri Lanka 26(3): 195-207. 